\title{
The predictor for Preoperative Coronal Imbalance in patients with Degenerative Lumbar Scoliosis-A related analysis on spinopelvic parameters
}

\section{Zifang ZHANG}

Nankai University

Dengbin QI

Chinese PLA General Hospital

\section{Tianhao WANG}

Chinese PLA General Hospital

\section{Zheng WANG}

Chinese PLA General Hospital

Yan WANG ( $\square$ yanwang301spine@163.com )

Chinese PLA General Hospital

\section{Research Article}

Keywords: degenerative lumbar scoliosis, coronal balance distance, coronal imbalance, L4 coronal tilt, L5 coronal tilt

Posted Date: February 19th, 2021

DOl: https://doi.org/10.21203/rs.3.rs-208773/v1

License: (1) This work is licensed under a Creative Commons Attribution 4.0 International License. Read Full License 


\section{Abstract}

Background: The mechanism of coronal imbalance is still unclear. The relationships among CBD and major curve, L4 and L5 tilt have been reported, which were not explicit although. The aim of this retrospective study was to explore and examine the association between spinopelvic parameters and coronal balance distance (CBD) in degenerative lumbar scoliosis (DLS) patients.

Methods Following institutional ethics approval, the radiographs of 161 DLS patients were retrospectively reviewed. The coronal measurements involving CBD, L4 coronal tilt, L5 coronal tilt, fractional curve, pelvic coronal tilt, the Cobb angle and included vertebras of major curve were documented. All of those participants were divided into group $A(C B D \geq 30 \mathrm{~mm})$ and group $B(C B D<30 \mathrm{~mm})$. Statistical analysis was performed to compare the difference of continuous and categorical variables. Pearson-Correlation, and Multiple-Regression Analysis were performed to explore the related parameter with CBD.

Results The CBD in 31 (19.25\%) DLS patients at pre-operation was over $30 \mathrm{~mm}$. With similar major Cobb angle, main curve of patients in the group $A$ had less involving vertebras $(P=0.009)$, but more rotation of the apical vertebra $(P<0.001)$ compared with that in the group $B$ respectively. Additionally, the $L 4$ coronal tilt in group $A$ was much larger $(P=0.02)$. Pearson-correlation analysis showed that $\mathrm{CBD}$ had significant relationship with $\mathrm{L} 4 / \mathrm{L} 5$ coronal tilt $(P<0.001)$, weak relationship with fractional curve $(P=0.027)$, but linearly independent with pelvic tilt and major Cobb. Stepwise Multiple- regression analysis revealed that only L4 tilt was the independent predictor for major Cobb and CBD.

Conclusions The prevalence of coronal imbalance at pre-operation in DLS patients is about $20 \%$. With similar major Cobb, the less the number of involving vertebra, the higher the incidence of coronal imbalance in DLS patients. L4 coronal tilt would be the predictive variable for progression of scoliosis and global coronal malalignment. Pelvis, the foundation of spine, had less effect on full-spine coronal balance.

\section{Key Points}

1. The prevalence of coronal imbalance in patients with degenerative lumbar scoliosis is common.

2. DLS patients with similar Cobb angle but less involving vertebras would be at the greatest risk for coronal imbalance at pre-operation.

3. L4 coronal tilt is an independent effective predictor for coronal imbalance, however, the foundation of spine, including fractional curve and pelvis, has less correlation with full-spine balance.

\section{Background}

Coronal imbalance is one of the major pathological symptom in patients with degenerative lumbar scoliosis(DLS ${ }^{[1]}$, which decreases health related quality of life (HRQOL) significantly ${ }^{[2,3]}$, and increases incidence of implant failure in patients performed long-fusion surgery ${ }^{[4]}$. According to the results of 
previous studies ${ }^{[5,6]}$, the incidence of coronal imbalance in those patients ranged from $19.3 \%$ to $34.8 \%$. The prevalence of this disorder is common, however, the pathophysiological mechanism of coronal imbalance is still unclear. There has been hypothesis that coronal imbalance might be brought by spinal stenosis, degeneration of discs and facet joints, or atrophy of paraspinal muscles, however, the hypothesis was short of solid evidence ${ }^{[5]}$. Pelvis plays an essential role on keeping full-body balance ${ }^{[7]}$. Additionally, previous study proposed that the ability to level the coronal tilt of L4 and L5 had greatest impact on the ability to achieve coronal balance ${ }^{[8]}$, but there was paucity of literature paying attention to analyze the relationship among coronal tilt of L4/L5, major curve, fractional curve, pelvic coronal tilt, and coronal balance distance(CBD).

The measurement of distance between $\mathrm{C7}$ plumb line(C7PL) and central sacral vertical line(CSVL), namely coronal balance distance(CBD) in this current study, has been widely used to reflect global coronal alignment. We performed this retrospective radiographic study in order to investigate the prevalence of coronal imbalance in DLS patients at pre-operation, and explore the relationships between radiological parameters and the coronal imbalance.

\section{Methods}

\section{Patients}

Approval by the Clinical Research Ethics Committee of our hospital was obtained before this research. We retrospectively documented radiographic data of DLS patients with preoperative standing full-spine Xrays in in our single institution from May 2015 to December 2018. General inclusion criteria for this study included any DLS patient $(\geq 45$ years old), diagnosed at our orthopedic department, who had integrated radiographic materials at baseline visit. Patients with prior spine surgery, spinal neoplasms, spinal tuberculosis, spinal or pelvic trauma, non-structural curvature, prior hip or knee surgery, or the discrepancy in leg length more than $2 \mathrm{~cm}$ were excluded from this study.

\section{Radiographic Evaluation}

Radiographic data collection consisted of full-length standing coronal and sagittal radiographs obtained in free-standing posture with the upper limbs resting on a support, the shoulders at $30^{\circ}$ forward flexion, and the elbows slightly flexed ${ }^{[9]}$. All of the radiographic parameters were measured with accurate and reliable spine measurement software (Surgimap version: 2.3.2.1; Spine Software, New York, NY) ${ }^{[10]}$.

On coronal radiographs 8 parameters were measured: 1) major Cobb angle, defined as the angle between the superior endplate of the most tilted vertebra cranially and the inferior endplate of the most tilted vertebra caudally (left scoliosis was recorded as negative[-], right scoliosis as positive[+]). 2) Lumbosacral fractional curve ${ }^{[11]}$, defined as the angle between the superior endplate of $L 4$ and the line formed by pedicles of S1 (left fractional curve was recorded as negative[-], right fractional curve as positive[+]). 3) Coronal balance distance(CBD) was defined as the horizontal distance between the C7PL and the center 
of the $\mathrm{S} 1$ on coronal plane, C7PL on the left side of CSVL was recorded as negative(-), right side as positive $\left.(+) \cdot{ }^{[12]} 4\right) L 4$ coronal tilt, defined as the angle between superior endplate of $L 4$ and the horizontal line(left side was recorded as negative[-], right side as positive[+]). $\left.{ }^{[5]} 5\right) \mathrm{L} 5$ coronal tilt, defined as the angle between superior endplate of L5 and the horizontal line(left side was recorded as negative[-], right side as positive $\left.[+]) .{ }^{[5]} 6\right)$ the number of involved vertebra in the structure curve. 7 ) the coronal pelvic tilt(C-PT), the angle between the line formed by iliac crests and the horizontal line(left side was recorded as negative[-], right side as positive[+]). 8) apical vertebra rotation by the Nash-Moe method(left side was recorded as negative[-], right side as positive[+] $)^{[13]}$. The detailed measured methods were shown in Figure $1 \mathbf{A}-\mathbf{B}$ and Figure 2.

\section{Statistical analysis}

Continuous variables with normal distribution are expressed as the Mean \pm standard deviation(SD) and abnormal data as the median. Categorical variables are expressed as counts and percentages. All of those radiographic data were measured with Surgimap by two independent researchers. Intra- and interrater reliabilities were excellent with kappa values ranging from 0.889 to 0.938 . Pearson correlation coefficient $(r)$ was calculated to test for associations between CBD and regional coronal parameters. Then, parameters having statistical relationship $(P<0.05)$ were further investigated using stepwise multiple regression analysis and the coefficient of determination $\left(R^{2}\right)$ was calculated. According to the recent criterion ${ }^{[14]}, 161$ patients were assigned into the coronal imbalance group $(C B D \geq 30.00 \mathrm{~mm}$, group A) and the coronal balance group $(\mathrm{CBD}<30.00 \mathrm{~mm}$, group $\mathrm{B})$. Those normal distributed variables were analyzed using independent-sample $t$ test, and analysis of abnormal distributed variables performed with Mann-Whitney U-test. Statistics were performed with SPSS 26.0 package software (version for Mac IBMã SPSSâ Statistics) with statistical significance set at $P<0.05$ (two-sided).

\section{Results}

\section{Group description}

A total of 161 patients including 132 men and 29 women met inclusion criteria with mean age: 63.88 years(SD 8.42). 106 patients had left side curve and right side curve appeared in the rest of 55 patients. There were no difference $(P>0.05)$ involving age, ratio of gender and types of curves between group $A$ and B.

\section{Radiographic results}

In this study, there were 31 patients with CBD over $30 \mathrm{~mm}$, and the incidence of coronal imbalance was $19.25 \%(31 / 161)$. Although major Cobb and the fractional curve had no differences respectively $(P>0.05)$ in two groups, the number of involving vertebra in major curve was less in group $A$ than that in group $\mathrm{B}(t=2.639, P=0.009)$, and the rotation of apical vertebra was much more $(t=-3.796, P<0.001)$. Moreover, the 
L4 coronal tilt was much more in group $A$ than that in group $B(t=1.992, P=0.02)$. The details were shown in table 1.

The mean coronal balance distance(CBD) was $0.14 \mathrm{~mm} \pm 20.5 \mathrm{~mm}$ (from $-57.6 \mathrm{~mm}$ to $68.2 \mathrm{~mm}$ ), the mean major Cobb was $-2.83^{\circ} \pm 26.83^{\circ}$ (from $-48.8^{\circ}$ to $61.7^{\circ}$ ), the mean fractional curve was

$3.18^{\circ} \pm 10.84^{\circ}$ (from $-33^{\circ}$ to $24.5^{\circ}$ ), the mean L4 coronal tilt was $3.77^{\circ} \pm 14.52^{\circ}$ (from $-22.9^{\circ}$ to $28.7^{\circ}$ ), the mean L5 coronal tilt was $1.91^{\circ} \pm 8.3^{\circ}$ (from $-16.2^{\circ}$ to $19.6^{\circ}$ ), and the mean pelvic tilt was $-0.19^{\circ} \pm 2.89^{\circ}$ (from $-6.7^{\circ}$ to $8.7^{\circ}$ ). The details were shown in table 2 .

\section{Relationship between CBD, the number of vertebra in major curve, major Cobb, fractional curve, L4/L5 coronal tilt, and pelvic tilt.}

Simple Line chart showed that the number of vertebra in major curve decreased with CBD increasing(Figure 3). Pearson correlation analysis showed CBD was significantly correlated with L4 $(r=-0.471, P<0.001)$ or L5 $(r=-0.468, P<0.001)$ coronal tilt, and weakly correlated with fractional curve $(r=-0.255, P=0.017)$. Although there were significant correlation between pelvic coronal tilt and regional parameters including major curve $(r=-0.366, P<0.001)$, L4 coronal tilt $(r=0.348, P<0.001)$, L5 coronal tilt $(r=0.351, P<0.001)$, and fractional curve $(r=0.552, P<0.001)$, neither major Cobb $(r=0.036, P=0.741)$ nor pelvic tilt $(r=0.206, P=0.056)$ had relationship with CBD. The details were shown in table 3.

\section{Regression Analysis}

On the basis of the results above, entry of regional parameters involving L4 coronal tilt, L5 coronal tilt, and fractional Cobb into stepwise multiple regression analysis showed that only L4 coronal tilt was the independent predictor of $\operatorname{CBD}\left(R^{2}=0.221, P<0.001\right)$, and the linear regression equation was obtained, $\mathrm{CBD}=0.583-0.742 * \mathrm{~L} 4$ tilt. Additionally, entry of major curve-related four variables including fractional curve, L4 coronal tilt, L5 coronal tilt, and coronal pelvic tilt into stepwise multiple regression analysis revealed that only L4 coronal tilt was an independent predictor of structure curve $\left(R^{2}=0.549\right.$, $P<0.001)$, and the linear regression equation was as follows: Major Cobb $=2.42-1.355 *$ L4 tilt. The scatter diagrams were shown in Figure 4 A-B.

\section{Discussion}

Spinal imbalance, involving the sagittal and/or coronal planes, is an essential index to the severity of spine deformity ${ }^{[15,16]}$. The increasing imbalance on the coronal plane in patients with degenerative lumbar scoliosis (DLS) has become one of main underlying factor that leads them to abnormal appearance, functional decline, and back pain ${ }^{[17]}$. The distance between $\mathrm{C} 7$ plumb line (C7PL) and central sacral vertical line (CSVL), named coronal balance difference ${ }^{[5]}$, coronal balance distance ${ }^{[12]}$, global coronal malalignment ${ }^{[14]}$, and $\mathrm{C} 7$ migration $^{[11]}$, has been used to reflect coronal alignment. In this current study, we defined the global coronal parameter as coronal balance distance (CBD). The latest study demonstrated the threshold of CBD was $30 \mathrm{~mm}$, after analyzing those related-variables involving the 
health-related quality of life (HRQOL), radiographic data, and complications in adult spinal deformity (ASD) patients ${ }^{[14]}$.

In our study, the prevalence rate of coronal imbalance was $19.25 \%(31 / 161)$ by the criterion of $30 \mathrm{~mm}$. According to previous studies ${ }^{[8,11]}$, structure curve had no influence on coronal imbalance in ASD patients. Comparisons in this current study revealed that although the degree of the main curvatures of patients in the two groups had no difference, the involving vertebras were less in patients with coronal imbalance (group A) than those with coronal balance (group B) $(t=2.639, P=0.009)$. Additionally, Line Chart shows the number of vertebras in the major curve decreases gradually with the mean value of CBD increasing. According to compensatory mechanism of spine ${ }^{[18]}$, the more the involved vertebras and the rotation degrees of apical vertebra, the larger the degrees of the structural curvatures, which can keep the global spinal balance. Contrarily, spine would be imbalance if the compensatory mechanism were broken. Therefore, according to the results in our study we deduce that DLS patients, with similar major Cobb angle, but less vertebras and more rotation degrees of those apical vertebras, would be at greater risk for coronal imbalance because of spinal decompensation.

Lewis et al. ${ }^{[8]}$ proposed that the coronal tilt of $L 4$ and $L 5$ were larger in ASD patients with CBD over $40 \mathrm{~mm}$, and the correction of coronal tilt of L4 and L5 had the greatest impact on restoration of global coronal balance, further prospective study involving larger number of participants is needed to verify that although. What's more, the threshold of CBD being as $30 \mathrm{~mm}$ would be more reasonable ${ }^{[14]}$. Bao et al. ${ }^{[19]}$ proposed that the horizontalization of L5 was the foundation of coronal spinal alignment, in terms of addressing lumbosacral fractional curve. A population of 161 patients with DLS were absorbed in our study. Comparisons of regional radiographic data between coronal balance and imbalance patients showed significant difference only in L4 coronal tilt. Pearson correlation analysis showed that there were significant relationship between $C B D$ and coronal tilt of $L 4$ and $L 5$, and weak relationship with fractional curve, however, only L4 coronal tilt was an independent predictor for CBD after multiple regression analysis. In other words, reconstruction of L4 coronal tilt would restore effectively the coronal imbalance in DLS patients.

Additionally, considering correction of main curve during long-fusion surgery in ASD patients, Zhang et al. ${ }^{[12]}$ suggested that there is significant correlation among pre-operative $C B D$, rectified Cobb angle of main curves, and post-operative CBD. Unfortunately, they did not explore the relationship between major curve and CBD further. The current study explore the relationships between major Cobb and those regional parameters involving L4/L5 coronal tilt, fractional curve, and coronal pelvic tilt, and the results showed significant relationship between each other. Moreover, L4 coronal tilt was independent predictor of major Cobb following stepwise multiple regression analysis. Therefore, the correction of L4 tilt would play the essential role on rectification of scoliosis and restoration of coronal balance for degenerative lumbar scoliosis patients.

Regarding to sagittal full-body balance in patients with spinal deformity, Ferrero et al. ${ }^{[7]}$ illustrated the key role of pelvis. Then, pelvis should be one of compensatory factor in global coronal balance. The results of 
this study revealed moderate relationship between coronal pelvic tilt and regional radiographic variables involving coronal tilt of L4/L5, major Cobb, and fractional curve, however, there was no relationship between pelvic tilt and CBD. Moreover, comparisons between balance and imbalance patients showed no difference of coronal pelvic tilt. In other words, pelvis would play less compensation on global coronal balance.

The limitation of this study should be mentioned. Although the number of patients was sufficient in this study, when all patients were divided into two groups, the patients in coronal imbalance group were much less(31 patients), and these may lead to bias. Furthermore, exploring the relationship between coronal balance distance and clinical findings, this current study did not consider those functional scores such as SRS-22 and ODI, and did not explore the role of those regional parameters on reconstruction surgery. Despite those limitations, we insist that the results from this study can be as the foundation for those purpose.

\section{Conclusion}

The prevalence of coronal imbalance in degenerative lumbar scoliosis(DLS) patients is about $20 \%$ at preoperation time. With similar major Cobb, the less the number of involving vertebra, the larger the CBD on DLS patients who would be at greater risk for coronal imbalance due to spinal decompensation. L4 coronal tilt would be an independent predictor on progression of structure curve and global coronal malalignment. Pelvis, the foundation of spine, had less effect on full-spine coronal balance.

\section{Abbreviations}

DLS, degenerative lumbar scoliosis; $\mathrm{CBD}$, coronal balance distance; HRQOL, health related quality of life; C7PL, C7 plumb line; CSVL, central sacral vertical line; MC, major Cobb; FC, fractional Cobb; CPT, coronal pelvic tilt; $M$, male; F, female; SD, standard deviation.

\section{Declarations}

1. Acknowledgements: Each author of this article certifies that the Chinese PLA general hospital approved the human protocol for this investigation and that all investigations were conducted in conformity with ethical principles of research. This work was performed at the Chinese PLA general hospital, Fuxing Road, Beijing, China.

2. There was no data published previously.

3. All data generated during this study are available from the corresponding author on reasonable request.

4. Competing Interest: The authors of this manuscript declare no competing interests.

5. Funding: All of those authors certify that neither they nor any member of their immediate family have funding or commercial association(Consultancies, stock ownership, equity interest, patent/licensing 
arrangements, etc.) that might pose a conflict of interest in connection with the submitting article.

6. Authors' contributions: ZHANG Z F wrote this article completely; ZHANG Z F \& QI D B measured and recorded all of the data for this article. WANG T H analyzed and interpreted the data. WANG Y \& WANG $Z$ designed and supervised this study. WANG $Z$ is the co-correspondence author for this article; QI D B is the co-first author for this article. All authors rad and approved this final manuscript.

7. This study was conducted with approval from the Ethics Committee of Chinese PLA General Hospital and was performed in accordance with the Declaration of Helsinki. Written informed consent to participate was obtained from all participants.

8. Acknowledgements: I appreciate sincerely for the support from my family.

\section{References}

1. Diebo BG, Shah NV, Boachie-Adjei O, et al. Adult spinal deformity. The Lancet. 2019;394(10193):160172.

2. Koller $\mathrm{H}, \mathrm{Pfanz} \mathrm{C}$, Meier $\mathrm{O}$, et al. Factors influencing radiographic and clinical outcomes in adult scoliosis surgery: a study of 448 European patients. Eur Spine J. 2016;25(2):532-48.

3. Acaroglu E, Guler UO, Olgun ZD, et al. Multiple Regression Analysis of Factors Affecting HealthRelated Quality of Life in Adult Spinal Deformity. Spine Deform. 2015;3(4):360-366.

4. Tanaka N, Ebata S, Oda K, et al. Predictors and Clinical Importance of Postoperative Coronal Malalignment After Surgery to Correct Adult Spinal Deformity. Clin Spine Surg. 2020;33(7):E337E341.

5. Bao H, Yan P, Qiu Y, et al. Coronal imbalance in degenerative lumbar scoliosis: Prevalence and influence on surgical decision-making for spinal osteotomy. Bone Joint J. 2016;98-B(9):1227-33.

6. Ploumis A, Simpson AK, Cha TD, et al. Coronal Spinal Balance in Adult Spine Deformity Patients With Long Spinal Fusions: A Minimum 2- to 5-Year Follow-up Study. J Spinal Disord Tech. 2015;28(9):3417.

7. Ferrero E, Liabaud B, Challier V, et al. Role of pelvic translation and lower-extremity compensation to maintain gravity line position in spinal deformity. J Neurosurg Spine. 2016;24(3):436-46.

8. Lewis SJ, Keshen SG, Kato S, et al. Risk Factors for Postoperative Coronal Balance in Adult Spinal Deformity Surgery. Global Spine J. 2018;8(7):690-697.

9. Marks $M$, Stanford C, Newton P. Which lateral radiographic positioning technique provides the most reliable and functional representation of a patient's sagittal balance? Spine (Phila Pa 1976). 2009;34(9):949-54.

10. Lafage R, Ferrero E, Henry JK, et al. Validation of a new computer-assisted tool to measure spinopelvic parameters. Spine J. 2015;15(12):2493-502.

11. Zhang J, Wang Z, Chi P, Chi C. Coronal T1 Pelvic Tilt, a Novel Predictive Index for Global Coronal Alignment in Adult Spinal Deformity. Spine (Phila Pa 1976). 2020;45(19):1335-1340. 
12. Zhang Z, Song K, Wu B, et al. Coronal Imbalance in Adult Spinal Deformity Following Posterior Spinal Fusion With Instrument: A Related Parameters Analysis. Spine (Phila Pa 1976). 2019;44(8):550-557.

13. Nash CL, Jr., Moe JH. A study of vertebral rotation. J Bone Joint Surg Am. 1969;51(2):223-9.

14. Buell TJ, Smith JS, Shaffrey $\mathrm{Cl}$, et al. Multicenter assessment of surgical outcomes in adult spinal deformity patients with severe global coronal malalignment: determination of target coronal realignment threshold. J Neurosurg Spine. 2020:1-14.

15. Xu L, Sun X, Huang S, et al. Degenerative lumbar scoliosis in Chinese Han population: prevalence and relationship to age, gender, bone mineral density, and body mass index. Eur Spine J. 2013;22(6):1326-31.

16. Wang G, Cui X, Jiang Z, et al. Evaluation and Surgical Management of Adult Degenerative Scoliosis Associated With Lumbar Stenosis. Medicine (Baltimore). 2016;95(15):e3394.

17. Heary RF. Evaluation and treatment of adult spinal deformity. Invited submission from the Joint Section Meeting on Disorders of the Spine and Peripheral Nerves, March 2004. J Neurosurg Spine. 2004;1(1):9-18.

18. Ploumis A, Transfledt EE, Denis F. Degenerative lumbar scoliosis associated with spinal stenosis. Spine J. 2007;7(4):428-36.

19. Bao H, Liu Z, Zhang Y, et al. Sequential correction technique to avoid postoperative global coronal decompensation in rigid adult spinal deformity: a technical note and preliminary results. Eur Spine J. 2019;28(9):2179-2186.

\section{Tables}

\begin{tabular}{|c|c|c|c|c|}
\hline & group $A \rrbracket n=31 \square$ & group $\mathrm{B} \square \mathrm{n}=130 \square$ & $\mathrm{t} / \mathrm{c}^{2}$ values & $P$ values \\
\hline Gender $\square \mathrm{M} \square \mathrm{F} \square$ & $8 \square 23$ & $21 \square 109$ & 1.579 & 0.205 \\
\hline Left $\llbracket$ Right & $22 \square 9$ & $84 \square 46$ & 0.449 & 0.536 \\
\hline Age(years) & $63.52 \pm 10.64$ & $63.96 \pm 7.84$ & 0.219 & 0.828 \\
\hline Major $\operatorname{Cobb}\left({ }^{\circ}\right)$ & $28.28 \pm 11.4$ & $26.66 \pm 9.84$ & -0.798 & 0.426 \\
\hline Apical rotation & $2.81 \pm 0.60$ & $2.32 \pm 0.77$ & -3.796 & $<0.001^{*}$ \\
\hline Fractional Curve $\left(^{\circ}\right)$ & $17.08 \pm 10.0$ & $14.4 \pm 6.25$ & -1.402 & 0.17 \\
\hline Vertebras & $3.87 \pm 0.85$ & $4.36 \pm 0.95$ & 2.639 & $0.009^{*}$ \\
\hline $\mathrm{CBD}(\mathrm{mm})$ & $41.03 \pm 11.83$ & $8.92 \pm 6.7$ & 14.532 & $<0.001^{*}$ \\
\hline L4 $\operatorname{tilt}\left({ }^{\circ}\right)$ & $16.29 \pm 5.59$ & $12.01 \pm 8.41$ & 1.992 & $0.02^{*}$ \\
\hline L5 $\operatorname{tilt}\left({ }^{\circ}\right)$ & $7.99 \pm 3.95$ & $6.59 \pm 5.24$ & 0.982 & 0.329 \\
\hline Pelvic tilt $\left({ }^{\circ}\right)$ & $2.52 \pm 2.15$ & $2.25 \pm 1.68$ & 0.554 & 0.581 \\
\hline
\end{tabular}

* $P<0.05 M$, male; $F$, female; $C B D$, coronal balance distance; $S D$, standard deviation. 


\begin{tabular}{lccc}
\hline \multicolumn{3}{l}{ Table 2. Mean Value and Range of parameters } \\
\hline Parameter & Mean Value & SD & Range \\
\hline CBD $(\mathrm{mm})$ & 0.14 & 20.49 & $-57.6-68.2$ \\
Major Cobb $\left({ }^{\circ}\right)$ & -2.83 & 26.82 & $-48.8-61.7$ \\
Fractional Cobb $\left({ }^{\circ}\right)$ & 3.18 & 10.83 & $-33-24.5$ \\
L4 tiltt $\left({ }^{\circ}\right)$ & 3.77 & 14.51 & $-22.9-28.7$ \\
L5 tilt $\left({ }^{\circ}\right)$ & 1.91 & 8.3 & $-16.2-19.6$ \\
Pelvic tilt $\left({ }^{\circ}\right)$ & -0.18 & 2.89 & $-6.7-8.7$ \\
\hline CBD, indicates coronal balance distance. & \\
\hline
\end{tabular}

\begin{tabular}{|c|c|c|c|c|c|c|}
\hline Variables & $\mathrm{CBD}(\mathrm{mm})$ & $\mathrm{MC}\left({ }^{\circ}\right)$ & $\mathrm{FC}\left({ }^{\circ}\right)$ & L4 tilt $\left({ }^{\circ}\right)$ & L5 $\operatorname{tilt}\left({ }^{\circ}\right)$ & $\mathrm{CPT}\left({ }^{\circ}\right)$ \\
\hline $\mathrm{CBD}(\mathrm{mm})$ & $\mathrm{X}$ & 0.036 & $-0.255^{*}$ & $-0.471^{\mathrm{D}}$ & $-0.468^{D}$ & 0.206 \\
\hline $\mathrm{MC}\left({ }^{\circ}\right)$ & & $\mathbf{X}$ & $-0.638^{D}$ & $-0.741^{D}$ & $-0.661^{D}$ & $-0.366^{\mathrm{D}}$ \\
\hline $\mathrm{FC}\left(\left(^{\circ}\right)\right.$ & & & $\mathbf{X}$ & $0.838^{\mathrm{D}}$ & $0.625^{\mathrm{D}}$ & $0.552^{\mathrm{D}}$ \\
\hline L4 $\operatorname{tilt}\left({ }^{\circ}\right)$ & & & & $\mathbf{X}$ & $0.837^{\mathrm{D}}$ & $0.348^{\mathrm{D}}$ \\
\hline L5 $\operatorname{tiltt}\left({ }^{\circ}\right)$ & & & & & $\mathbf{X}$ & $0.351^{\mathrm{D}}$ \\
\hline $\mathrm{CPT}\left({ }^{\circ}\right)$ & & & & & & $\mathrm{X}$ \\
\hline
\end{tabular}

Note: CBD, indicate coronal balance distance; MC, major Cobb; FC, fractional Cobb; CPT, coronal pelvic tilt. *, $P<0.05$ (two-tailed); D, $P<0.001$ (two-tailed).

\section{Figures}




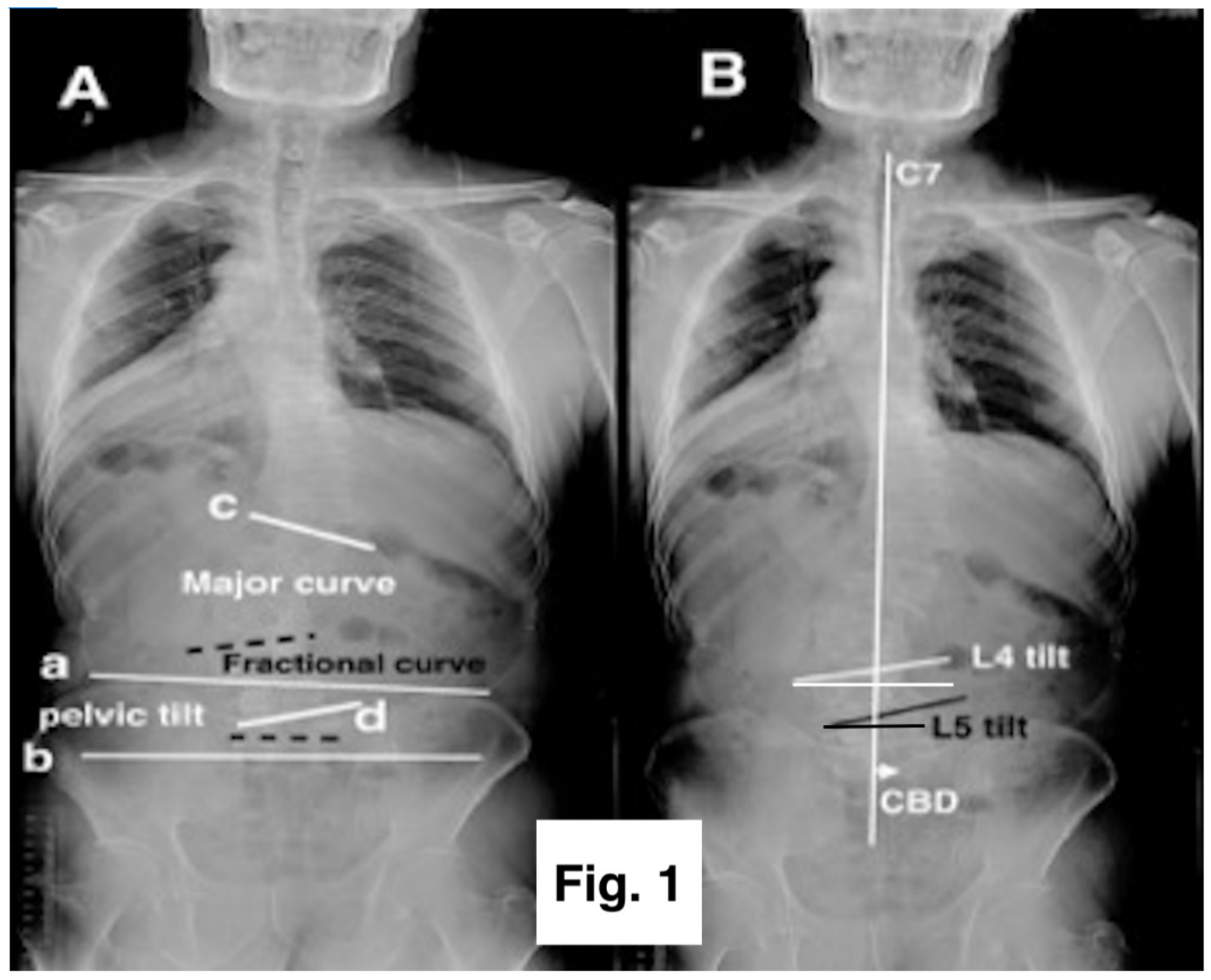

\section{Figure 1}

Schematic drawing of each parameter. Major curve, Coronal pelvic tilt, Fractional curve, (A). Coronal balance distance(CBD), L4 coronal tilt, and L5 coronal tilt.(B) 


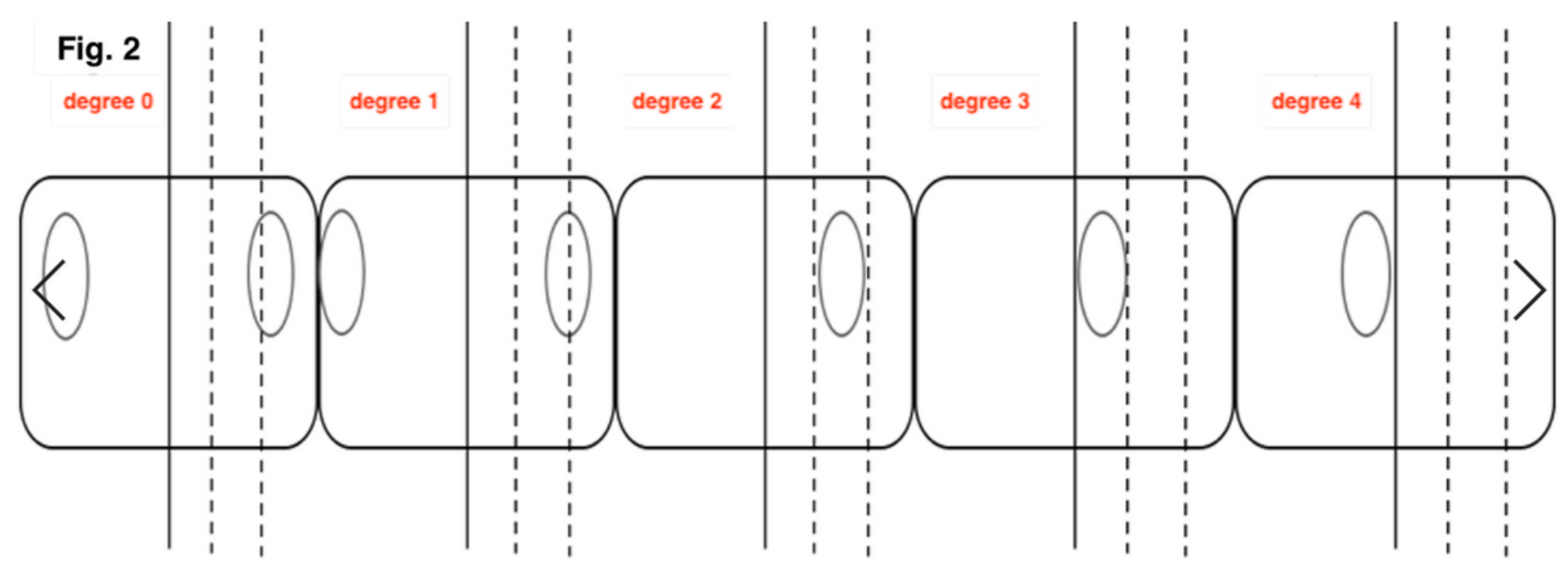

Figure 2

Nash-Moe method categorizes vertebral rotation into five degree. According to this method, the vertebra is first bisected longitudinally and then each half is further divided into 3 equal portions. No significant vertebral rotation exists when the distance from the vertebral pedicle shadow to the bilateral edges of the vertebral body is equal, indicating that the Nash-Moe grade is 0 . There is significant vertebral rotation when the vertebral pedicle shadow on the concave side is closer to the edge than that on the convex side, or disappears completely. Grade 1 is defined when most of the vertebral pedicle shadow on the convex side is still within the one-third of the edge portion; grade 2 is defined when it is within the one-third of the central portion; grade 3 is refined when it is within to one-third portion close to the midline; grade 4 is defined when it exceeds the midline. 


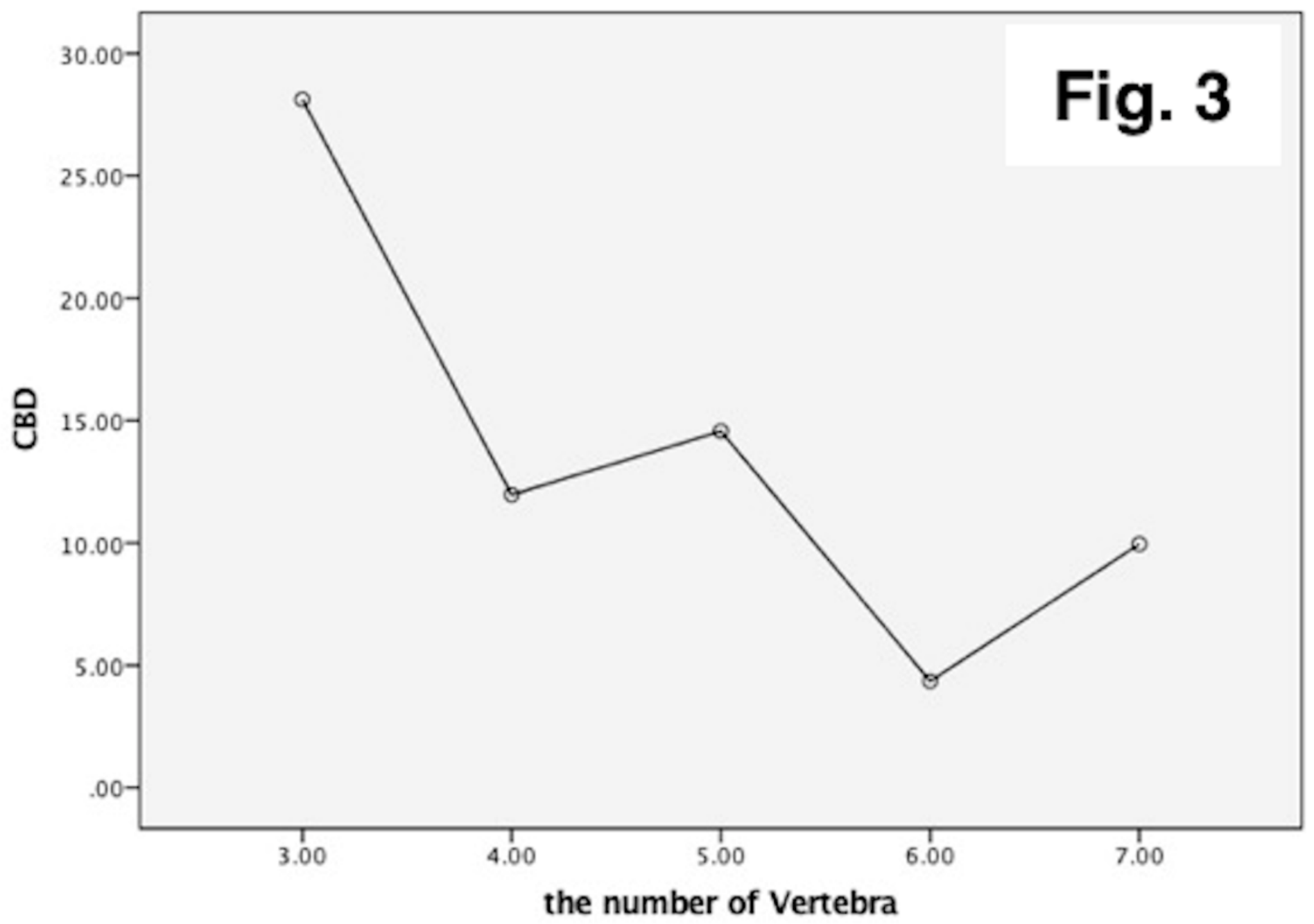

Figure 3

Association trend between coronal balance distance(CBD) and the number involving in Major curve.
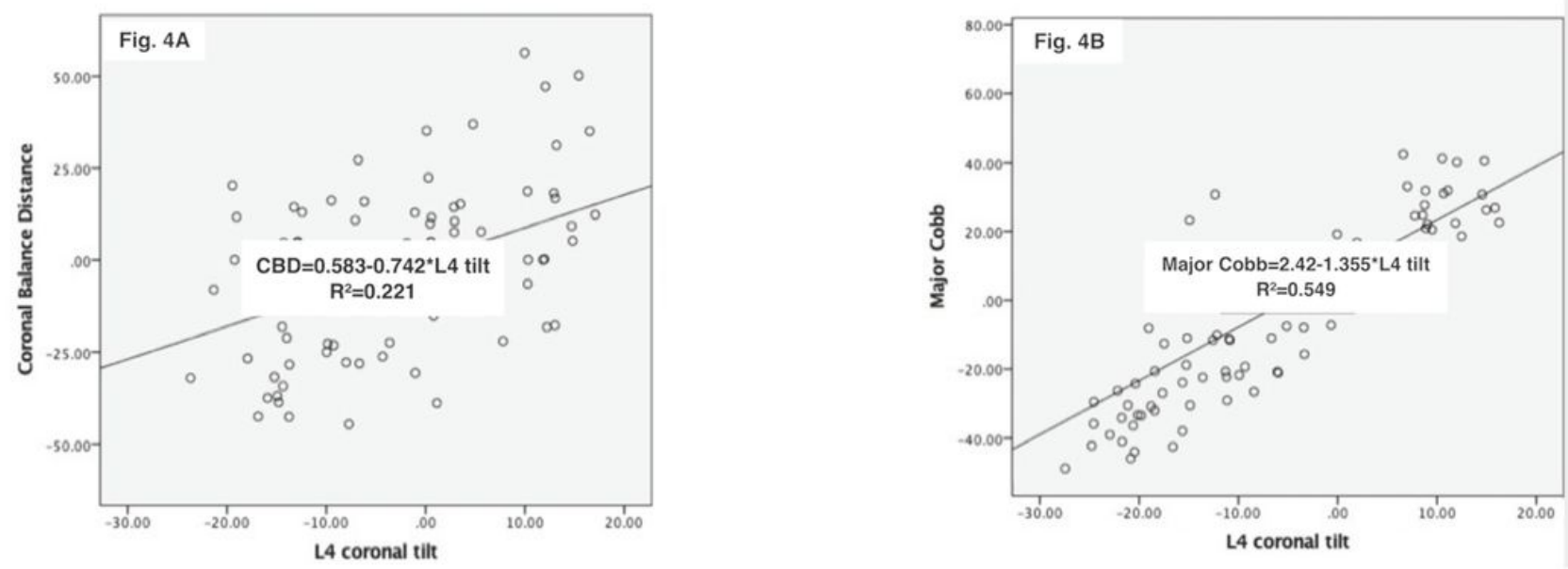

Figure 4 
Association between coronal balance distance(CBD) and $L 4$ coronal tilt $(A)$, and that between major curve and L4 coronal tilt (B). 